\title{
Chronological changes in the expression of phosphorylated tau and 5-AMP-activated protein kinase in the brain of senescence-accelerated P8 mice
}

\author{
HAK-SU KIM ${ }^{1,2}$, SOHEE MOON ${ }^{1,2}$, SUJIN KIM $^{3}$, MIN-JAE LEE ${ }^{3}$, MIN HWA SUK ${ }^{4}$, \\ DONG-HO PARK ${ }^{3}$, DONG WUN SHIN ${ }^{5}$, CHANG-SHIN PARK ${ }^{1,2}$ and JU-HEE KANG ${ }^{1,2}$
}

\author{
${ }^{1}$ Department of Pharmacology and Medicinal Toxicology Research Center; ${ }^{2}$ Hypoxia-Related Diseases Research Center, \\ Inha University School of Medicine; ${ }^{3}$ Department of Kinesiology, College of Arts and Sports, Inha University, \\ Incheon 22212; ${ }^{4}$ Department of Physical Education, College of Education, Seoul National University, Seoul 08826; \\ ${ }^{5}$ Department of Emergency Medicine, Inje University Ilsan Paik Hospital, Goyang, Gyeonggi 10380, Republic of Korea
}

Received February 27, 2016; Accepted February 10, 2017

DOI: $10.3892 / \mathrm{mmr} .2017 .6361$

\begin{abstract}
Senescence-accelerated mouse prone 8 (SAMP8), a non-transgenic animal model used for researching sporadic Alzheimer's disease (AD), presents AD pathologies and overall dysregulation in brain energy metabolism, which is one of the early pathogenic characteristics of AD. In the present study, the authors examined chronological changes in the expression patterns of phosphorylated tau and of proteins related to energy metabolism to evaluate the association of tau phosphorylation and metabolism, using young-(2-months-old), middle(5-months-old) and old-aged (10-months-old) SAMP8. The levels of phosphorylated 5'-AMP activated protein kinase at Thr172 (p-AMPK) and phosphorylated glycogen synthase kinase $3 \beta\left(\mathrm{p}-\mathrm{GSK} 3 \beta_{\mathrm{S} 9}\right)$ in the cortex of SAMP8 at 2 months were significantly higher than those in senescence-accelerated mouse resistant 1 (SAMR1). The differences were not detected at 5 and 10 months of age, which were concurrent with the changes in levels of phosphorylated tau at Ser396 (p-tau s396 $)$, but not with $\mathrm{p}-\mathrm{tau}_{\mathrm{S} 262}$. The level of $\mathrm{p}-\mathrm{tau}_{\mathrm{S} 262}$ was considerably higher in the cortex of middle-aged SAMP8 when compared with that of SAMR1 and sustained in old-aged SAMP8, but not in the young cortex. The levels of cortical sirtuin1 (Sirt1) and insulin receptor substrate 1 (IRS-1) expression of young SAMP8 were significantly lower, when compared with those in SAMR1. However, in the hippocampus of SAMP8, the patterns of chronological changes and levels of p-tau, p-AMPK, Sirt1 and IRS-1 relative to SAMR1 were different from those in the
\end{abstract}

Correspondence to: Dr Ju-Hee Kang, Department of Pharmacology and Medicinal Toxicology Research Center, Inha University School of Medicine, Inha University, 100 Inha-ro, Yonghyeon-dong, Nam-gu, Incheon 22212, Republic of Korea E-mail: johykang@inha.ac.kr

Key words: senescence-accelerated mice, Alzheimer's disease, tau, AMP-activated protein kinase, glycogen synthase kinase $3 \beta$ cortex. Taken together, the results suggested that regulation of tau phosphorylation via the AMPK-GSK3 $\beta$ pathway concurrent with dysregulation of energy metabolism may precede the pathological tau hyperphosphorylation in the cortex of SAMP8, and that the regulation of AMPK-GSK3 $\beta$-mediated tau phosphorylation may be dependent on phosphor-epitope in tau or the region of brain.

\section{Introduction}

Senescence-accelerated mice prone (SAMP) strains, which have been developed through the selective inbreeding of the AKR/J strain, present a senescence-related phenotype involving a short lifespan and rapid progression of senescence. Together with SAMP strains, senescence-accelerated mice resistant (SAMR) strains have been developed as their corresponding controls. Of the nine SAMP sub-strains, the mice of P8 strain (SAMP8) exhibit Alzheimer's disease (AD)-like pathologies, such as abundant expression of amyloid precursor protein (APP) and production of amyloid-beta, tau hyperphosphorylation and increased oxidative stress (1-4). In addition, the neural network activity and number of neurons in the brain of SAMP8 spontaneously reduce; this relates to their cognitive dysfunction $(1,2)$. To identify the mechanisms of AD and develop effective therapeutic strategies, appropriate animal models are essential. Although studies using SAMP8 are expanding, as SAMP8 mimics the non-familial form of AD compared to transgenic AD models, the relationship between tau phosphorylation and metabolic dysfunction in SAMP8 remains unclear.

Insulin and insulin-like growth factor-1 (IGF-1) signaling are associated with aging and control vital growth, survival, longevity and energy metabolism in the brain (5-7). A defect of insulin/IGF-1 signaling is associated with neurofibrillary tangles (NFTs) and amyloid plaque in $\operatorname{AD}(5,8)$ and administration of insulin can improve memory and cognitive function in patients with AD (9). Dysregulation of insulin/IGF-1 signaling may be mediated by insulin receptor substrate type 1 (IRS-1), which is a key mediator of the signaling pathway, and IRS-1 is 
dysregulated in the AD brain. In fact, reduced levels of IRS-1 have been detected in neurons in the medial temporal cortex of AD, and the inhibitory phosphorylation of IRS-1 at Ser312 and Ser616 is increased in neurons with NFTs (10). The relationship between impaired insulin actions and AD pathogenesis is widely accepted (11).

AMP-activated protein kinase (AMPK), a key regulator of cellular energy homeostasis, serves a critical role in neuronal survival $(12,13)$. Previous studies have indicated that the pathogenesis of neurodegenerative diseases such as AD may involve the alteration of AMPK signaling. For example, the activation of AMPK by pharmacological activators increases $A \beta$ production and tau hyper-phosphorylation (14-16). Conversely, studies demonstrating that AMPK activation inhibits $A \beta$ abundance and tau phosphorylation at several sites have been reported (17-21). Moreover, AMPK activation may be beneficial to reduce the neurotoxicity of glucose deprivation, A $\beta$ or $\alpha$-synuclein (22-24). Collectively, the role of AMPK in the pathogenesis of AD, such as tau phosphorylation and A $\beta$-mediated neurotoxicity, remains controversial.

Previous studies indicated that impaired glucose homeostasis is observed in the SAMP8 strain along with higher levels of glucose, insulin and free fatty acids in serum $(25,26)$. Moreover, inhibited activity of AKT and expression of glucose transporter 4 in the skeletal muscle in the SAMP8 strain, when compared to the SAMR1 control strain, has been reported (26). In a previous SAMP8 study of the authors, there was observation of AMPK activity upregulation in the cortex of young SAMP8 (pre-symptomatic age), when compared with SAMR1 mice; a change attributable to the inhibition of tau phosphorylation at Ser396 (27). However, the chronological relationship between metabolic regulators and AD pathogenesis, particularly in tau hyper-phosphorylation in SAMP8, has not been fully elucidated. However, it has been reported that AD pathologies are developed in SAMP8 as early as 4-5 months of age (3).

The aim of the present study was to characterize the tau phosphorylation and the expression of proteins related to energy metabolism in order to understand tau-related pathogenesis and energy metabolism. In order to accomplish this, the authors evaluated chronological changes in the expression of phosphorylated tau and intracellular metabolic regulators, including AMPK, GSK3 $\beta$, sirtuin1 (Sirt1) and IRS-1, in the brains of SAMP8 across different ages [young (2-month-old), middle (5-month-old) and old (10-month-old)] to examine both pre-symptomatic and symptomatic stages of AD.

\section{Materials and methods}

Animal care. The care of animals was conducted according to the Guide for Care and Use of Laboratory Animals of the National Institutes of Health (NIH, Bethesda, MD, USA). All experiments were conducted in accordance with procedures and protocols approved by the Animal Care and Handling Committee of Inha University (Incheon, Korea). Male SAMR1 ( $n=30$; body weight: $25-29 \mathrm{~g})$ and SAMP8 $(n=30$; body weight: 24-29 g) (all six-weeks-old) were obtained from Central Laboratory Animal, Inc. (Seoul, Korea). Mice were housed individually in a temperature- $\left(22 \pm 2^{\circ} \mathrm{C}\right)$ and humidity-controlled (45-55\%) room under a $12 \mathrm{~h}$ light-dark cycle (7:00 a.m.-7:00 p.m.), with free access to a standard rodent diet and water. Mice ( $n=10$, each strain) were sacrificed at 2, 5 and 10 months of age $(n=20$, at each time point).

Isolation of the hippocampus and cerebral cortex. Under intraperitoneal ketamine $(80 \mathrm{mg} / \mathrm{kg})$ and xylazine $(8 \mathrm{mg} / \mathrm{kg}$ )-induced anesthesia, the skin was flipped over the eyes to free the skull, and an incision was made at the top of the skull, beginning at the caudal part through the anterior part of the skull, in order to expose the brain. The brain was quickly isolated and the cerebral hemispheres were separated by a sagittal midline incision following removal of the cerebellum. Using forceps, the diencephalon was carefully removed to expose the medial side of the hippocampus. From the remaining hemisphere containing the cortex and hippocampus, the C-shaped hippocampus was dissected out using fine scissors and a spatula. The whole cerebral cortex and dissected hippocampus were immediately frozen in liquid nitrogen, and stored at $-80^{\circ} \mathrm{C}$ until use.

Immunoblotting. To prepare tissue lysates, the collected hippocampi or cerebral cortices of SAMR1 and SAMP8 mice were ground in radioimmunoprecipitation assay buffer (Sigma-Aldrich; Merck KGaA, Darmstadt, Germany) using a pellet pestle followed by homogenization under sonication. The debris of the homogenates was removed by centrifugation at $13,000 \times \mathrm{g}$ for $15 \mathrm{~min}$ at $4^{\circ} \mathrm{C}$, and total protein content of the supernatant was quantified using a bicinchoninic acid assay protein assay kit (Pierce; Thermo Fisher Scientific, Inc., Waltham, MA, USA). Samples were prepared with dithiothreitol (Sigma-Aldrich; Merck KGaA) and denatured by heating at $95^{\circ} \mathrm{C}$ for $3 \mathrm{~min}$. Proteins $(10-20 \mu \mathrm{g})$ were separated by $4-20 \%$ SDS-PAGE gels and transferred to polyvinylidene difluoride membranes in a transfer buffer containing $25 \mathrm{mM}$ Tris-HCl, 192 mM glycine and 10\% methanol. Membranes were blocked with $5 \%$ BSA or 5\% non-fat dry milk in TBS with $0.1 \%$ Tween-20 (Sigma-Aldrich; Merck KGaA) and incubated with specific primary antibodies against AMPK (2532; 1:1,000; Cell Signaling Technology, Inc., Danvers, MA, USA), p-AMPK at Thr172 (p-AMPK; 2535; 1:1,000; Cell Signaling Technology, Inc.), phosphorylated Acetyl-CoA carboxylase at Ser79 (p-ACC; 3661; 1:1,000; Cell Signaling Technology, Inc.), insulin receptor substrate 1 (IRS-1; 06-248; 1:1,000; Upstate, Biotechnology, Inc., Lake Placid, NY, USA), tau (Tau5; SIG-39413; 1:1,000; Covance, Inc., Princeton, NJ, USA), phosphorylated tau at Ser262 (p-tau ${ }_{\mathrm{S} 262}$; sc-32828; 1:500; Santa Cruz Biotechnology, Inc., Dallas, TX, USA), phosphorylated tau at Ser396 (p-tau ${ }_{\mathrm{S} 396}$; sc-12414; 1:1,000; Santa Cruz Biotechnology, Inc.), sirtuin-1 (Sirt1; 9475; 1:1,000; Cell Signaling Technology, Inc.), $\alpha$-synuclein (C-20; sc-7011; 1:1,000; Santa Cruz Biotechnology, Inc.), phosphorylated GSK3 $\beta$ at Ser9 (p-GSK3 $\beta_{\text {S9 }}$; 9336, 1:1,000; Cell Signaling Technology, Inc.), GSK3 $\beta$ (sc-9166, 1:1,000; Santa Cruz Biotechnology, Inc.) at $4^{\circ} \mathrm{C}$ for overnight, or $\beta$-actin (a1978, 1:10,000, Sigma-Aldrich; Merck KGaA) at room temperature for $2 \mathrm{~h}$, and then incubated with appropriate secondary antibodies at room temperature for $2 \mathrm{~h}$ (peroxidase conjugated anti-rabbit IgG; NCI1460KR; 1:5,000 and peroxidase conjugated anti-mouse IgG; NCI1430KR; 1:5,000; both from Thermo Fisher Scientific Inc.). Immunoreactive bands 
were visualized by an enhanced chemiluminescence detection system (15,159; Pierce; Thermo Fisher Scientific, Inc.) and quantified using the image analysis software Quantity One (version 4.6.6; Bio-Rad Laboratories, Inc., Hercules, CA, USA).

Statistical analysis. Results are expressed as the mean \pm the standard error of the mean, unless otherwise stated. $\mathrm{P}<0.05$ was deemed statistically significant using GraphPad Prism version 5.0 (GraphPad Software Inc., La Jolla, CA, USA). The Mann-Whitney nonparametric test was used to compare groups.

\section{Results}

Characteristics of tau phosphorylation in the cerebral cortex of SAMP8. In order to employ the SAMP8 strain as an appropriate experimental model for understanding the precise molecular mechanisms of $\mathrm{AD}$, a profiling analysis through a time course study of proteins related to AD pathogenesis was necessary. To characterize AD-related tau phosphorylation, the authors initially investigated the expression levels of tau phosphorylation at the positions of both the KXGS motif (i.e.,

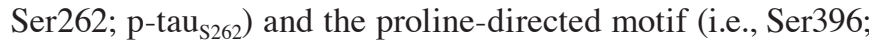
$\left.\mathrm{p}-\mathrm{tau}_{\mathrm{S} 396}\right)$ in the cerebral cortices and hippocampi obtained from SAMP8 or SAMR1 at young (2-months-old), middle (5-months-old) and old (10-months-old) ages. In the cerebral cortex of SAMP8, differential levels of p-tau ${ }_{\mathrm{S} 262}$ and p-tau $\mathrm{S}_{\mathrm{S} 366}$ were observed, compared to those in SAMR1. The lower level of $\mathrm{p}-\mathrm{tau}_{\mathrm{S} 396}$ in the cortex of 2-month-old SAMP8 compared to SAMR1 controls increased in a time-dependent manner up to 10-months-old, at which point it reached levels comparable the SAMR1 controls ( $\mathrm{P}<0.05$; Fig. $1 \mathrm{~A}$ and $\mathrm{B})$. Meanwhile, the level of $\mathrm{p}-\mathrm{tau}_{\mathrm{S} 262}$ in young SAMP8 was comparable to that in SAMR1; however, these levels significantly increased in the cortex of middle-aged SAMP8 and were sustained in old SAMP8, when compared to those of age-matched SAMR1 $(\mathrm{P}<0.05$; Fig. 1A and $\mathrm{C})$. The chronological changes in the cortical level of $\mathrm{p}$-tau S396 $_{39}$ were negatively correlated with the expression of phosphorylated GSK3 $\beta$ ( $\mathrm{P}<0.05$; Fig. 1A and D). In addition, the pattern of changes in the level of active (phosphorylated at Thr172) AMPK, an upstream kinase of GSK $3 \beta$ and ACC, was similar to GSK3 $\beta_{\mathrm{S} 9}$ and $\mathrm{p}$-tau $\mathrm{Ta}_{\mathrm{S} 396}$ (Fig. 3A and E). There are several reports that tau pathology can be accelerated by overexpression of $\alpha$-synuclein $(28,29)$. However, the expression level of $\alpha$-synuclein in the cortex of SAMP8 was not significantly different from that of SAMR1 ( $\mathrm{P}>0.05$; Fig. $1 \mathrm{~A}$ and $\mathrm{E})$ at all ages.

Characteristics of hippocampal tau phosphorylation of SAMP8. In the hippocampus, $\mathrm{p}-\mathrm{tau}_{\mathrm{S} 396}$ and $\mathrm{p}-\mathrm{tau}_{\mathrm{S} 262}$ were observed to increase as SAMP8 increased in age (Fig. 2A-C). In young SAMP8, a significant difference was not observed between the expressions of $\mathrm{p}$-tau at both sites $(\mathrm{P}>0.05)$, however, the levels of hippocampal p-tau in aged SAMP8 were higher than age-matched SAMR1 ( $\mathrm{p}-\operatorname{tau}_{\mathrm{S} 262}, \mathrm{P}<0.01$; Fig. 2A and C). The level of hippocampal p-GSK3 $\beta_{\mathrm{S} 9}$ expression in young SAMP8 was significantly higher than in SAMR1 $(\mathrm{P}<0.01$; Fig. 2D), but the pattern of changes in expression levels of $\mathrm{p}-\mathrm{tau}_{\mathrm{S} 396}$ and $\mathrm{p}-\mathrm{AMPK}$ was not similar. Similar to the cortex, the expression levels of $\alpha$-synuclein and total tau in the hippocampus of SAMP8 were not significantly different from that in the hippocampus of SAMR1 (P>0.05; Fig. 2A and E).

Changes in the expression of proteins related to aging and cellular metabolism in the cortex of SAMP8. The authors demonstrated the chronological expression pattern of proteins related to senescence (Sirt1) and intracellular energy metabolism (AMPK and IRS-1) in the brains of SAMP8. In the cerebral cortex of SAMP8, the level of Sirt1 expression at 2-months-old was significantly lower than in SAMR1 controls $(\mathrm{P}<0.001)$, which persisted until 5-months-old and disappeared in mice at 10-months-old (Fig. 3A and B). Interestingly, the level of IRS-1 expression in the cortex of young SAMP8 was significantly lower than in SAMR1, which persisted until 10-months-old $(\mathrm{P}<0.05$ at 2 months, $\mathrm{P}<0.001$ at 5 and 10 months; Fig. 3A and $\mathrm{C}$ ). In addition, the levels of active p-AMPK and of inhibitory phosphorylation of ACC at Ser79, a well-known AMPK substrate, in the cerebral cortex of young SAMP8 were significantly higher than those in SAMR1 (both $\mathrm{P}<0.05$ ), with the increase disappearing in middle- and old-aged mice (Fig. 3A, D and E).

Changes in the expression of proteins related to aging and cellular metabolism in the SAMP8 hippocampus. In the hippocampus of SAMP8, the level of Sirt1 expression at young age was significantly lower, when compared with levels in SAMR1 ( $\mathrm{P}<0.05$; Fig. 4A and B). However, the levels of Sirt1 expression in old SAMP8 and SAMR1 were not significantly different. Finally, the levels of other proteins (IRS-1, p-AMPK and $\mathrm{p}-\mathrm{ACC}_{\mathrm{S} 79}$ ) in SAMP8 were not significantly different from those in SAMR1 ( $\mathrm{P}>0.05$; Fig. 4A, C-E) across all ages, which was different from the pattern of changes of these proteins in the cortex.

\section{Discussion}

Multiple mechanisms have been implicated in the development of $\mathrm{AD}$, including accumulation of $\mathrm{A} \beta$, oxidative stress, increased synaptic damage, hyperphosphorylation of tau, defective neurogenesis and alterations in the signal transduction pathways associated with neuronal survival (1-4). Therefore, a number of studies have been conducted to augment the information of SAMP8 as a model of AD using the aforementioned targets (2,30). Apart from these mechanisms, the present study investigated whether the brains of SAMP8 that mimic the $\mathrm{AD}$ brain present dysregulation of molecules involved in both energy metabolism and tau phosphorylation (i.e., AMPK and GSK $3 \beta$ ), and whether the alteration of AMPK-GSK3 $\beta$ activity is related to the hyperphosphorylation of tau.

In terms of energy metabolism, the brain largely relies on circulating glucose as a primary source of fuel because it stores only small sources of energy as glycogen; therefore, understanding glucose metabolism in the brain is important $(11,13)$. Moreover, type 2 diabetes increases the risk of sporadic AD, and AD leads to insulin resistance, which is associated with dysfunction of IRS-1 and Sirt1 in the brain $(10,31)$. Consistent with previous studies (32), the current study observed an early and significant decrease in both IRS-1 and Sirt1 expression in the cortex of SAMP8. A double knockout of IRS-1 and -2 
A

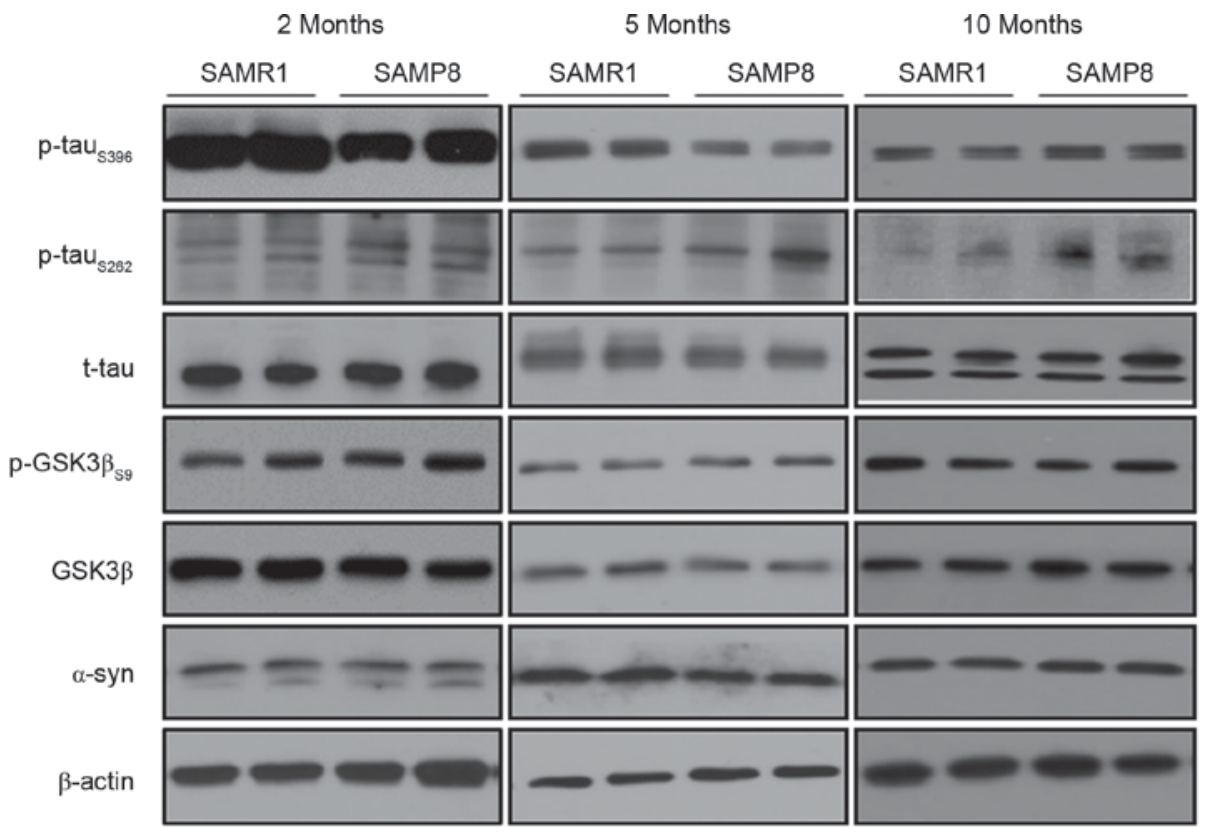

B

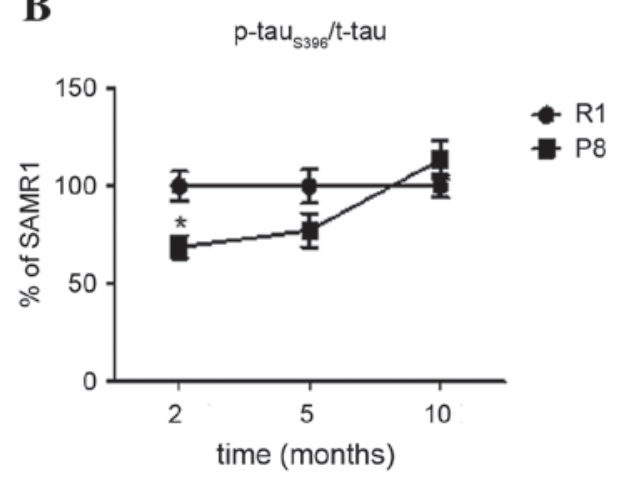

D

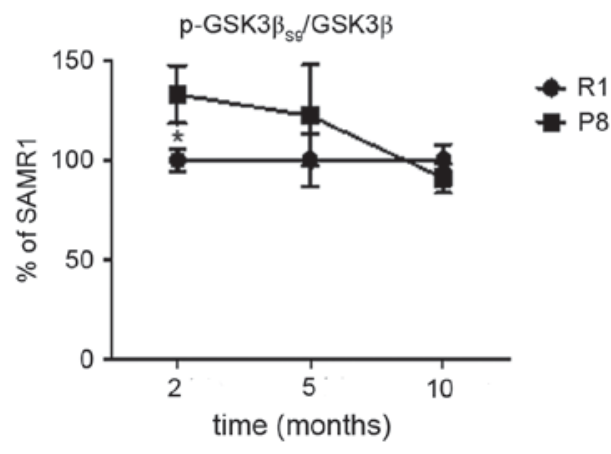

$\mathbf{C}$

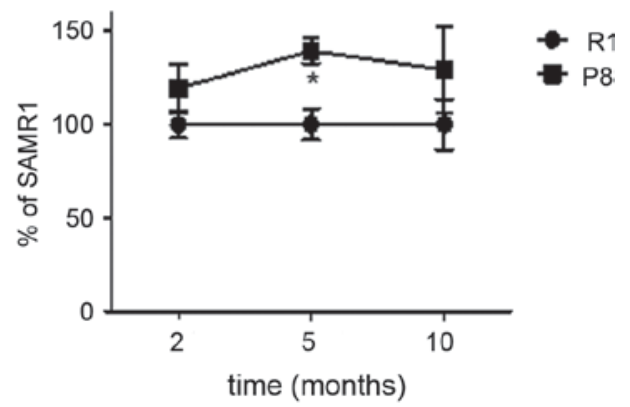

$\mathbf{E}$

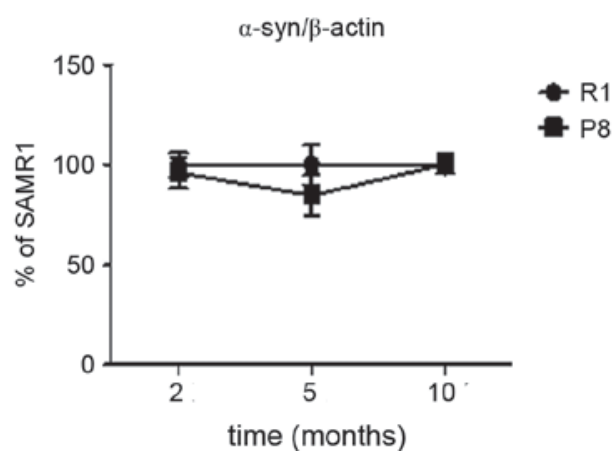

Figure 1. Expression levels of phosphorylated tau ( $\mathrm{p}-\mathrm{tau}_{\mathrm{S} 396}$ and $\mathrm{p}$-tau $\left.\mathrm{S}_{\mathrm{S} 262}\right), \mathrm{p}-\mathrm{GSK} 3 \beta_{\mathrm{S} 9}$ and $\alpha$-syn in the cerebral cortex of SAMP8 or SAMR1 at different ages using immunoblotting. (A) Representative blots of proteins from the cerebral cortex are presented. The mean expression levels of $(\mathrm{B}) \mathrm{p}$-tau $\mathrm{S}_{\mathrm{S} 96},(\mathrm{C}) \mathrm{p}$-tau $\mathrm{S}_{\mathrm{S} 262}$ and (D) p-GSK3 $\beta_{\mathrm{s} 9}$, normalized using their total form of proteins, are depicted as being compared with age-matched SAMR1 controls. (E) The mean relative expression levels of $\alpha$-syn, compared between the two mouse strains ( $n=10$ each). $\mathrm{P}<0.05$ vs. age-matched SAMR1 controls using the Mann-Whitney U test. $\alpha$-syn, $\alpha$-synuclein; GSK3 $\beta$, glycogen synthase kinase $3 \beta$; SAMP8, senescence-accelerated mouse prone 8 (P8); SAMR1, senescence-accelerated mouse resistant $1(\mathrm{R} 1)$.

in the skeletal muscle has demonstrated the activation of AMPK (33), and Sirt1 and AMPK also serves roles in the energy-sensing network that controls energy expenditure and protects against metabolic imbalance in various cells including neurons (34). The authors identified significantly reduced levels of IRS-1 and Sirt1 in the cortex of SAMP8 mice, which was accompanied by increased AMPK activity at young ages. Since the Sirtl pathway is closely related to AMPK signaling as a sensor of energy availability $(35,36)$, the authors speculated that the reduction of IRS-1 and Sirt1 expression may involve the compensatory activation of AMPK in the cortex of young SAMP8 mice. It is not clear why the association of 
A

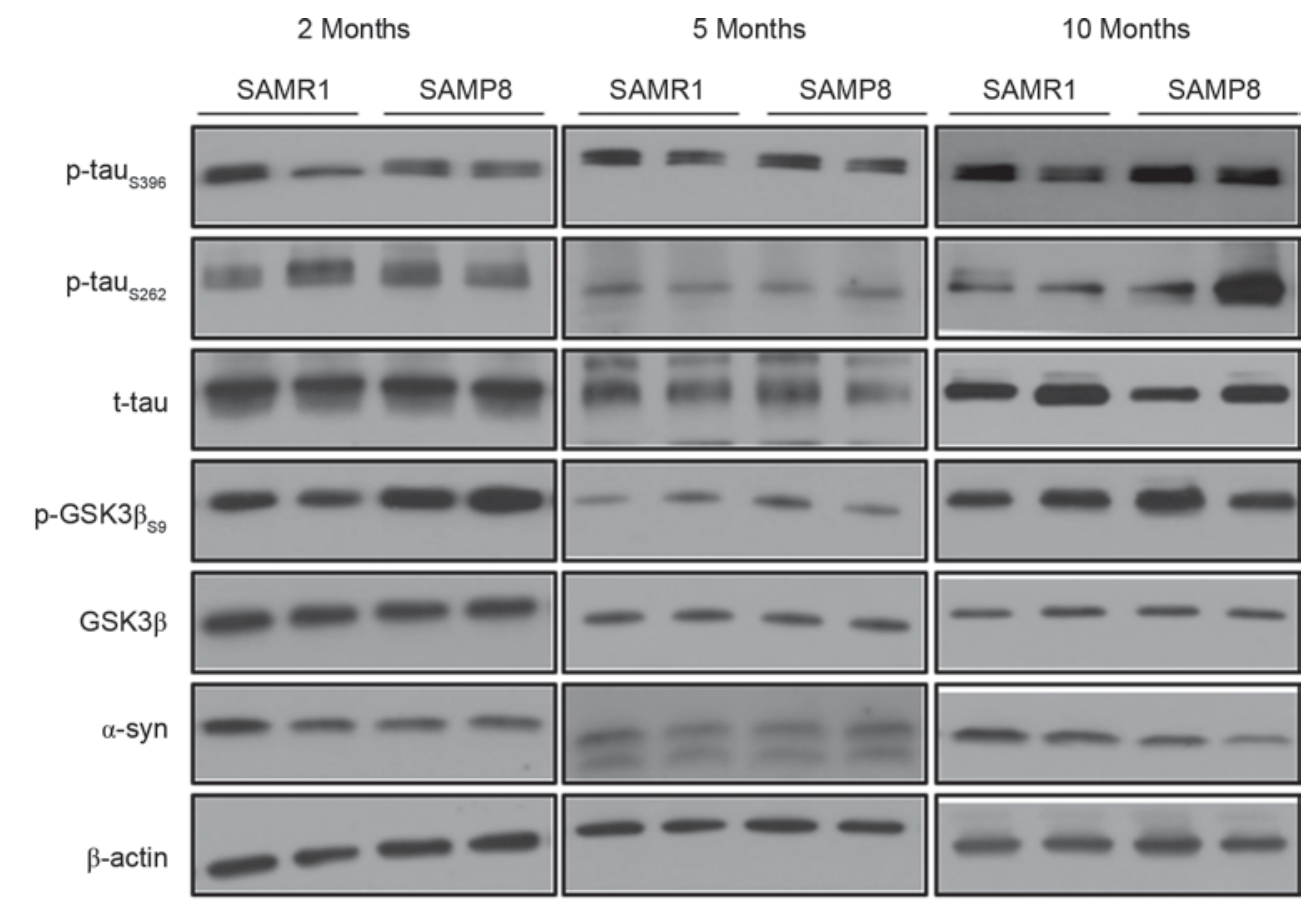

B

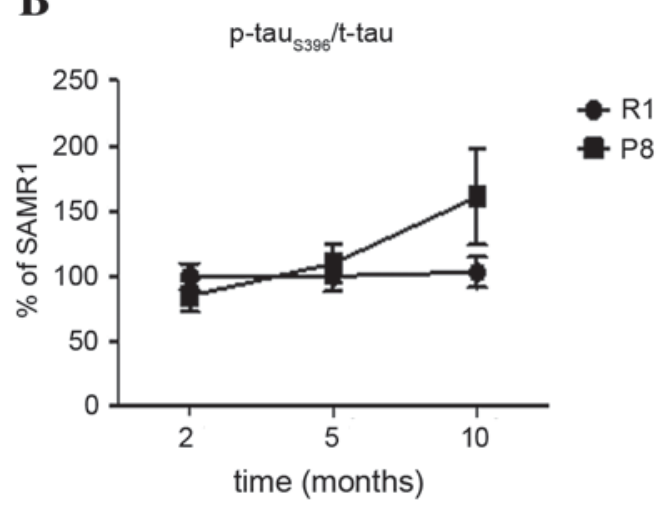

D

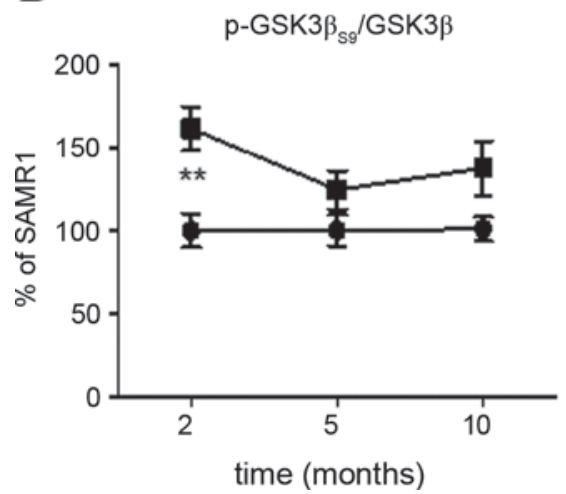

$\mathbf{C}$

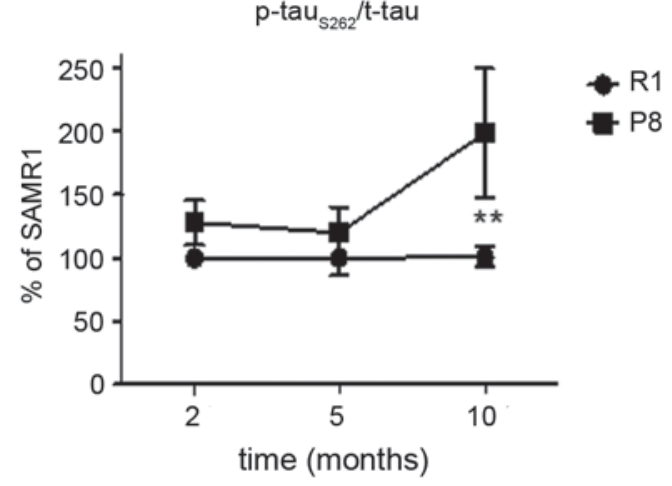

$\mathbf{E}$

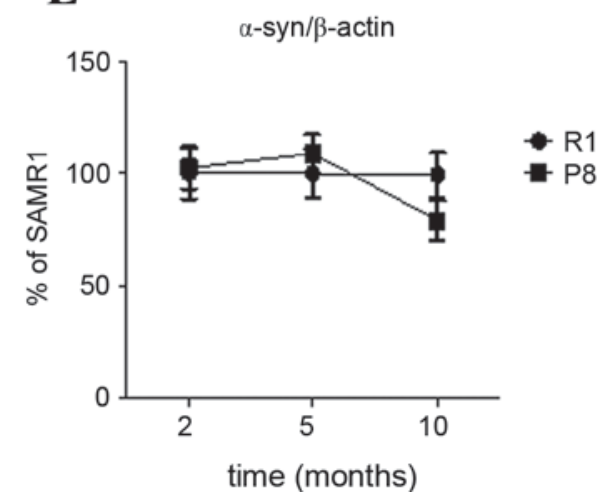

Figure 2. Expression levels of phosphorylated tau ( $\left(\mathrm{t}^{-\mathrm{tau}_{\mathrm{S} 396}}\right.$ and $\left.\mathrm{p}-\mathrm{tau}_{\mathrm{S} 262}\right), \mathrm{p}-\mathrm{GSK} 3 \beta_{\mathrm{s} 9}$ and $\alpha$-syn in the hippocampus of SAMP8 or SAMR1 across different ages. (A) Representative blots for proteins from the hippocampus are presented. The mean expression levels of (B) p-tau $\mathrm{S}_{\mathrm{S} 396}$, (C) p-tau $\mathrm{s}_{\mathrm{s} 262}$ and (D) p-GSK3$\beta_{\mathrm{S} 9}$, normalized by their total form of proteins, are depicted as being compared with age-matched SAMR1 controls. (E) The mean relative expression levels of $\alpha$-syn, compared between the two mouse strains ( $n=10$ each). ${ }^{* *} \mathrm{P}<0.01$ vs. age-matched SAMR1 controls using the Mann-Whitney U test. GSK3 $\beta$, glycogen synthase kinase 3 $\beta$; $\alpha$-syn, $\alpha$-synuclein; SAMP8, senescence-accelerated mouse prone 8 (P8); SAMR1, senescence-accelerated mouse resistant 1 (R1).

IRS-1 and Sirt1 expression with AMPK disappeared in older SAMP8; it may be that the prolonged suppression of insulin signaling pathways disturbed the compensatory activation of AMPK $(35,36)$.
AMPK has recently been proposed as a novel therapeutic target for regulating metabolism-related pathogenesis in $\mathrm{AD}$, as well as a target in $\mathrm{A} \beta$ - and tau-related pathogenesis. AMPK inhibits the activity of GSK $3 \beta$ through increasing the 


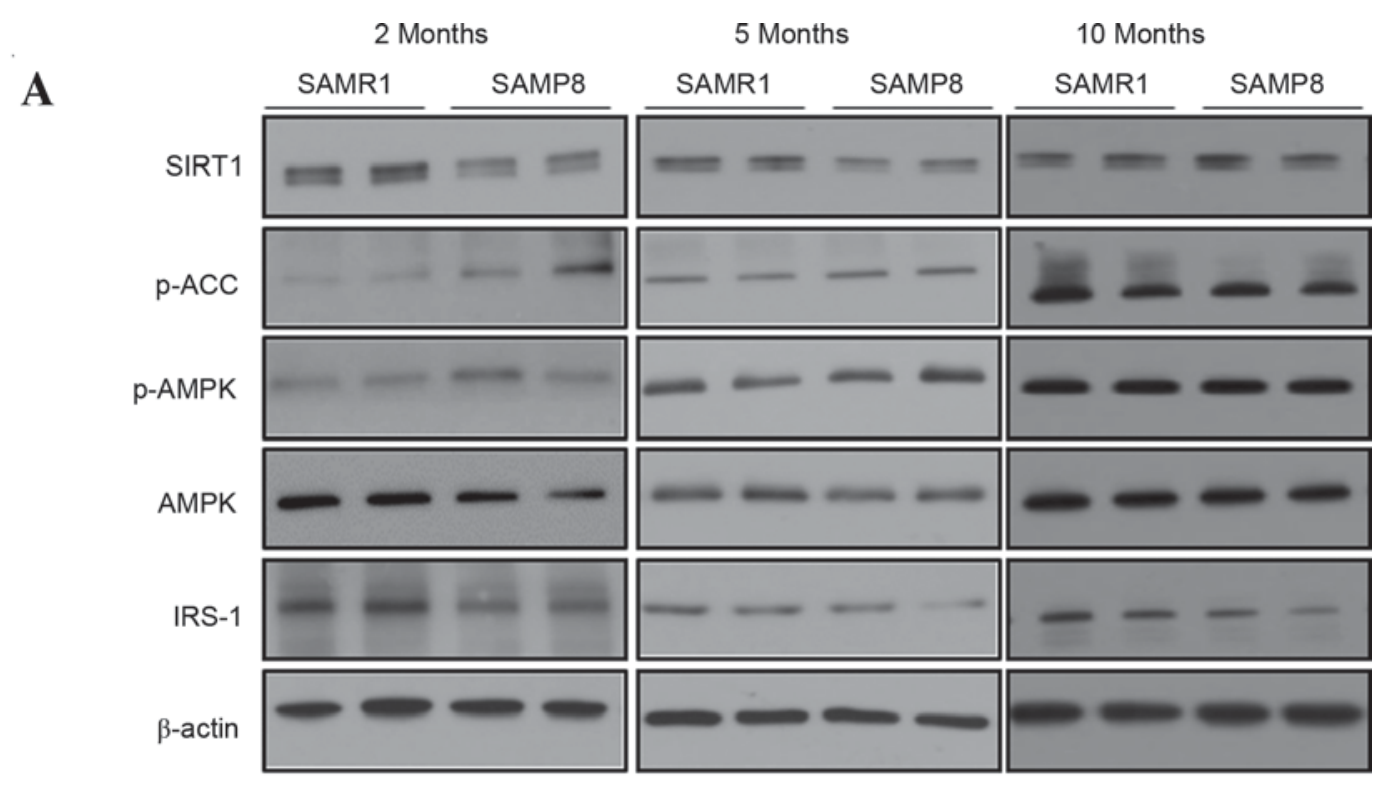

B

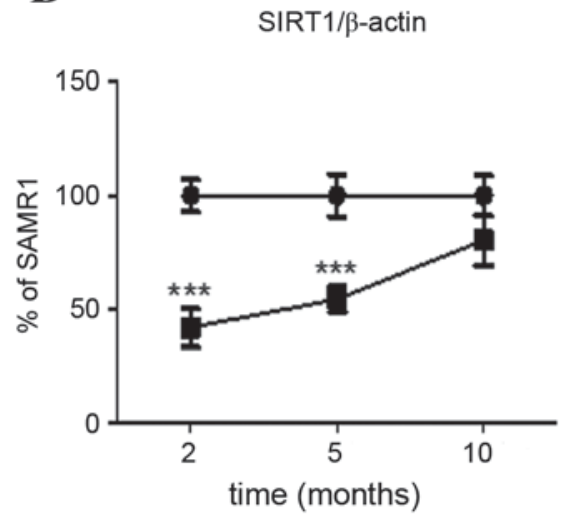

D

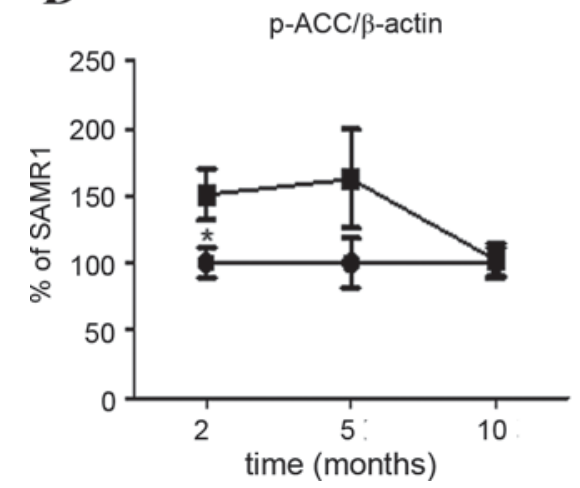

C

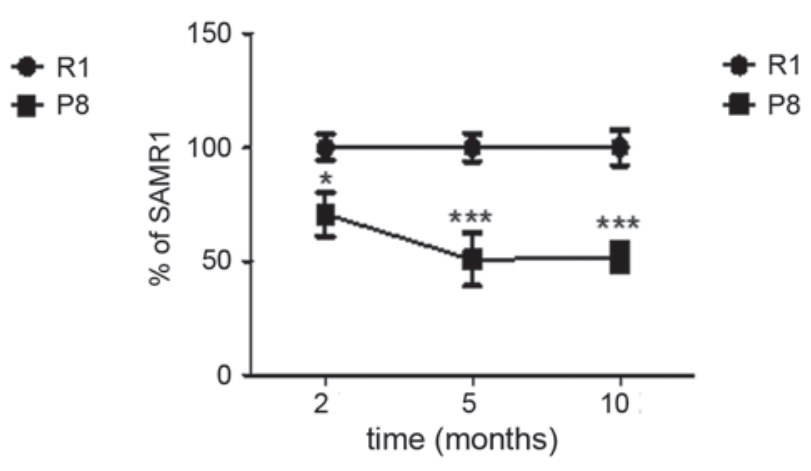

$\mathbf{E}$

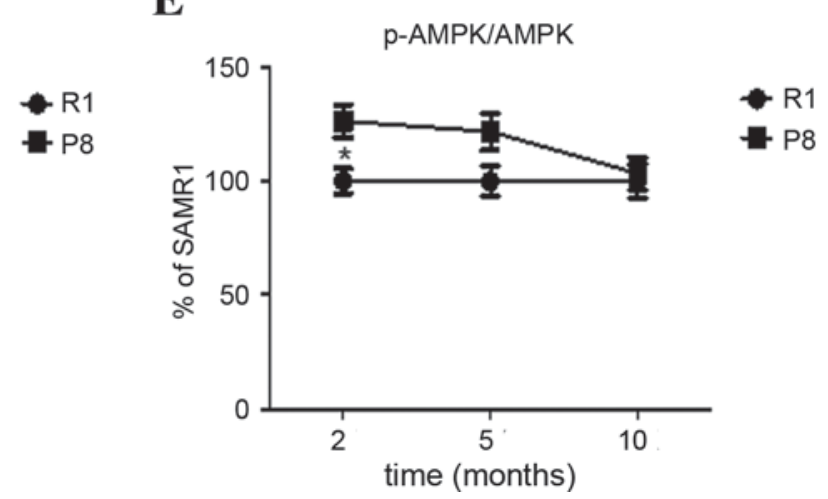

Figure 3. Expression levels of Sirt1, IRS-1 and p-ACC and p-AMPK in the cerebral cortex of young-, middle- and old-aged SAMP8 or SAMR1. (A) Representative immunoblots of proteins from the cerebral cortex are presented. The mean expression levels of (B) Sirt1, (C) IRS-1 and (D) p-ACC of SAMP8 were compared with those in age-matched SAMR1 controls. (E) The activity of AMPK (relative expression levels of p-AMPK to total AMPK) compared across ages (n=10 each). ${ }^{*} \mathrm{P}<0.05 ;{ }^{* * *} \mathrm{P}<0.001$ vs. age-matched SAMR1 controls using the Mann-Whitney U test. Sirt1, sirtuin1; IRS-1, insulin receptor substrate 1; ACC, acetyl-CoA carboxylase; AMPK, AMP-activated protein kinase; SAMP8, senescence-accelerated mouse prone 8 (P8); SAMR1, senescence-accelerated mouse resistant $1(\mathrm{R} 1)$.

inhibitory phosphorylation of GSK3 $\beta$ at Ser9 (37). GSK3 $\beta$ is a well-known tau kinase that generates pathological phosphor-epitopes at proline-directed sites, such as p-tau ${ }_{3396}$, in tau, which has been described as a reliable marker of AD (37-39). Accordingly, the authors hypothesized that AMPK activation may inhibit GSK3 $\beta$ activity, resulting in a reduction of $p-\operatorname{tau}_{\mathrm{S} 396}$ in young SAMP8. This hypothesis was supported by evidence that the pattern of p-AMPK in the cortex of SAMP8 at 2,5 and 10 months of age was correlated with the levels of p-GSK3 $3 \beta_{\mathrm{s} 9}$ and negatively correlated with the levels of $\mathrm{p}-\mathrm{tau}_{\mathrm{S} 396}$. Moreover, a previous study of the authors indicated that 5-aminoimidazole-4-carboxamide ribonucleotide-induced AMPK 
A

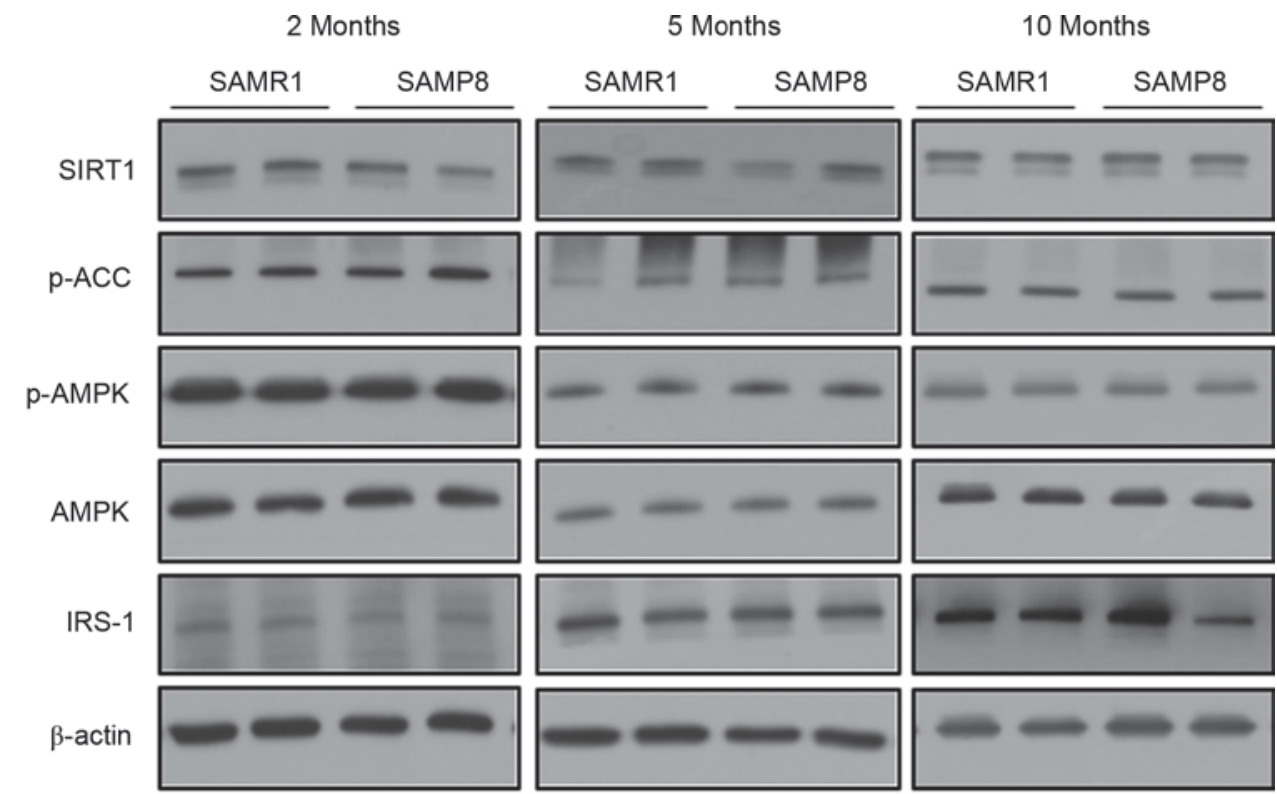

$\mathbf{B}$

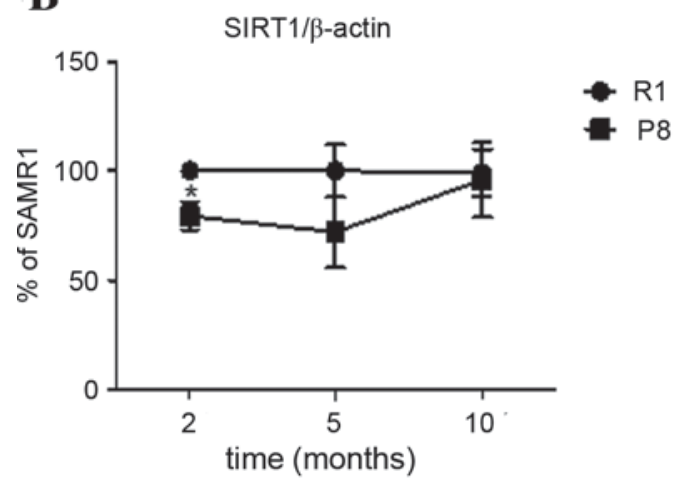

D

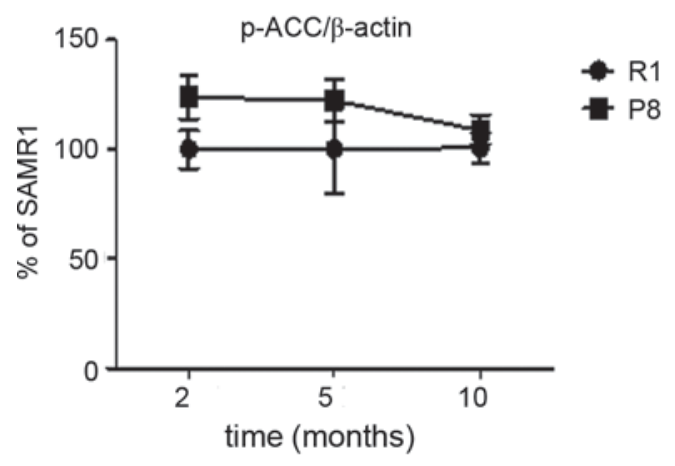

C

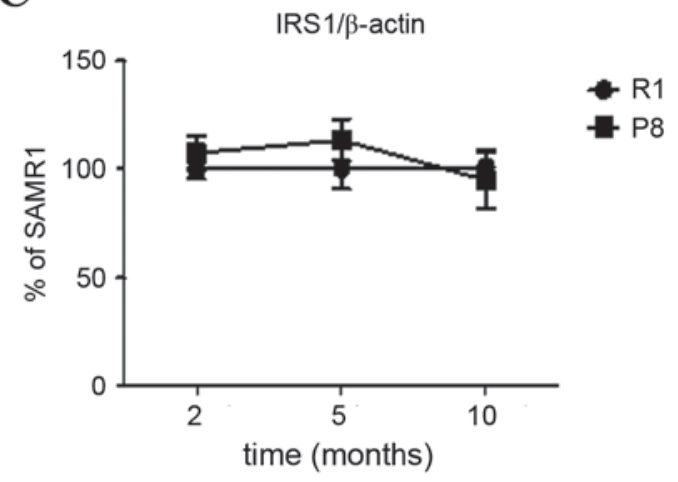

$\mathbf{E}$

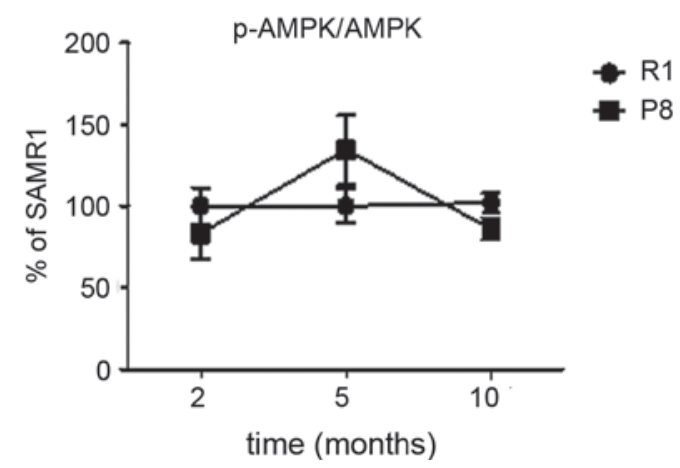

Figure 4. Expression levels of Sirt1, IRS-1 and p-ACC and p-AMPK in the hippocampus of young-, middle- and old-aged SAMP8 or SAMR1. (A) Representative immunoblots of proteins from the cerebral cortex are presented. The mean expression levels of (B) Sirt1, (C) IRS-1 and (D) p-ACC of SAMP8 were compared with those in age-matched SAMR1 controls. (E) The activity of AMPK (relative expression levels of p-AMPK to total form of AMPK) compared across ages (n=10 each). "P<0.05 vs. age-matched SAMR1 controls using the Mann-Whitney U test. Sirt1, sirtuin1; IRS-1, insulin receptor substrate 1; ACC, acetyl-CoA carboxylase; AMPK, AMP-activated protein kinase; SAMP8, senescence-accelerated mouse prone 8 (P8); SAMR1, senescence-accelerated mouse resistant 1 (R1).

activation reduces GSK3 $\beta$ activity and levels of $\mathrm{p}^{-\operatorname{tau}_{\mathrm{S} 396}}$ in differentiated SH-SY5Y cells (27). Thus, these data suggested that the activity of AMPK in the cerebral cortex of young SAMP8 was associated with decreased levels of p-tau ${ }_{5396}$ via a reduction of GSK $3 \beta$ activity. In addition, a significant increase to $\mathrm{p}$-tau $\mathrm{S}_{\mathrm{S} 22}$ levels in the cortex of middle-aged SAMP8 mice was observed, when compared to those of age-matched
SAMR1 mice. However, the pattern of changes in cortical levels of p-tau ${ }_{\mathrm{S} 262}$ were not similar to the changes in activity of AMPK and GSK $3 \beta$. Neither were the chronological changes in levels of p-tau ${ }_{\mathrm{S} 396}$ and $\mathrm{p}$-tau $\mathrm{Su}_{\mathrm{S} 262}$ in the hippocampus similar to the changes in the activity of AMPK and GSK $3 \beta$. Collectively, the role of GSK $3 \beta$ and AMPK in tau phosphorylation may be dependent on the phosphor-epitopes (e.g., p-tau ${ }_{\mathrm{S} 396}$ and 
$\mathrm{p}$ - $\mathrm{tau}_{\mathrm{S} 262}$ ) in tau or the region (e.g., cortex and hippocampus) of SAMP8 brain. In neuronal cells, AMPK impairs dendritic growth and branching of primary hippocampal neurons (40). Several studies have recently revealed that AMPK activation protects neuronal cells from cell death triggered by abnormalities in brain energy metabolism or accumulation of $A \beta$ or $\alpha$-synuclein $(23,24,41)$. Therefore, whether AMPK activation is beneficial or harmful at the preclinical stage of AD should be further evaluated in various in vivo models and in clinical studies.

Animal models are extremely useful to predict the outcome of drug candidates for prevention or therapy of $\mathrm{AD}$, as well as to study mechanistic hypotheses for AD pathogenesis. However, no animal model recapitulates the entirety of AD pathophysiology in humans. Therefore, it is important to characterize the advantages and limitations of particular animal models. Among the animal models of AD, the sequence of pathologies and clinical characteristics of SAMP8 is similar to that observed in human patients with AD (2). In the present study, the data provided basic information on SAMP8 that included the relationship between alterations in energy metabolism and AD-related tau hyper-phosphorylation in the brain. Based on these data, the authors have suggested that the SAMP8 strain of mice has pathologic similarities to AD, and is therefore an excellent model for advancing the knowledge for AD-related metabolic dysfunction through age-related neurodegenerative processes. However, it should be noted that selected sites of the phosphor-epitope in tau may be differentially regulated by the AMPK-GSK $3 \beta$-mediated pathway, and particular regions of the brain may be a factor in studies targeting tau phosphorylation in the SAMP8 AD model.

In conclusion, the presented results demonstrated early deteriorations in energy metabolism and concurrent alterations of tau hyper-phosphorylation dependent on the region of the brain and phosphor-tau epitopes. In addition, these findings provided basic information on the chronological changes in the expression of metabolic proteins and AD-related tau phosphorylation in SAMP8, and this model may allow researchers to further investigate preventive and therapeutic targets for AD.

\section{Acknowledgements}

This study was financially supported by Mid-career Researcher Program (grant no. 2013R1A2A2A01008223) and Medical Research Center (grant no. 2014009392) through the National Research Foundation of Korea (NRF).

\section{References}

1. Takeda T: Senescence-accelerated mouse (SAM): A biogerontological resource in aging research. Neurobiol Aging 20: 105-110, 1999.

2. Morley JE, Armbrecht HJ, Farr SA and Kumar VB: The senescence accelerated mouse (SAMP8) as a model for oxidative stress and Alzheimer's disease. Biochim Biophys Acta 1822: 650-656, 2012.

3. Morley JE, Kumar VB, Bernardo AE, Farr SA, Uezu K, Tumosa N and Flood JF: Beta-amyloid precursor polypeptide in SAMP8 mice affects learning and memory. Peptides 21: 1761-1767, 2000.

4. Canudas AM, Gutierrez-Cuesta J, Rodriguez MI, Acuña-Castroviejo D, Sureda FX, Camins A and Pallàs M: Hyperphosphorylation of microtubule-associated protein tau in senescence-accelerated mouse (SAM). Mech Ageing Dev 126: 1300-1304, 2005.
5. de la Monte SM and Tong M: Brain metabolic dysfunction at the core of Alzheimer's disease. Biochem Pharmacol 88: 548-559, 2014.

6. Aberg ND, Brywe KG and Isgaard J: Aspects of growth hormone and insulin-like growth factor-I related to neuroprotection, regeneration, and functional plasticity in the adult brain. ScientificWorldJournal 6: 53-80, 2006.

7. Kleinridders A, Ferris HA, Cai W and Kahn CR: Insulin action in brain regulates systemic metabolism and brain function. Diabetes 63: 2232-2243, 2014.

8. Cole GM and Frautschy SA: The role of insulin and neurotrophic factor signaling in brain aging and Alzheimer's Disease. Exp Gerontol 42: 10-21, 2007.

9. Reger MA, Watson GS, Frey WH II, Baker LD, Cholerton B, Keeling ML, Belongia DA, Fishel MA, Plymate SR, Schellenberg GD, et al: Effects of intranasal insulin on cognition in memory-impaired older adults: modulation by APOE genotype. Neurobiol Aging 27: 451-458, 2006.

10. Moloney AM, Griffin RJ, Timmons S, O'Connor R, Ravid R and O'Neill C: Defects in IGF-1 receptor, insulin receptor and IRS-1/2 in Alzheimer's disease indicate possible resistance to IGF-1 and insulin signalling. Neurobiol Aging 31: 224-243, 2010.

11. Biessels GJ and Reagan LP: Hippocampal insulin resistance and cognitive dysfunction. Nat Rev Neurosci 16: 660-671, 2015.

12. Hardie DG: AMPK-sensing energy while talking to other signaling pathways. Cell Metab 20: 939-952, 2014.

13. Weisová P, Dávila D, Tuffy LP, Ward MW, Concannon CG and Prehn JH: Role of 5'-adenosine monophosphate-activated protein kinase in cell survival and death responses in neurons. Antioxid Redox Signal 14: 1863-1876, 2011.

14. Chen Y, Zhou K, Wang R, Liu Y, Kwak YD, Ma T, Thompson RC, Zhao Y, Smith L, Gasparini L, et al: Antidiabetic drug metformin (GlucophageR) increases biogenesis of Alzheimer's amyloid peptides via up-regulating BACE1 transcription. Proc Natl Acad Sci USA 106: 3907-3912, 2009.

15. Vingtdeux V, Davies P, Dickson DW and Marambaud P: AMPK is abnormally activated in tangle- and pre-tangle-bearing neurons in Alzheimer's disease and other tauopathies. Acta Neuropathol 121: 337-349, 2011.

16. Thornton C, Bright NJ, Sastre M, Muckett PJ and Carling D: AMP-activated protein kinase (AMPK) is a tau kinase, activated in response to amyloid beta-peptide exposure. Biochem $\mathrm{J} 434$ : 503-512, 2011.

17. Vingtdeux V, Chandakkar P, Zhao H, d'Abramo C, Davies P and Marambaud P: Novel synthetic small-molecule activators of AMPK as enhancers of autophagy and amyloid- $\beta$ peptide degradation. FASEB J 25: 219-231, 2011.

18. Cai Z, Li B, Li K and Zhao B: Down-regulation of amyloid- $\beta$ through AMPK activation by inhibitors of GSK-3 $\beta$ in SH-SY $5 Y$ and SH-SY5Y-AßPP695 cells. J Alzheimers Dis 29: 89-98, 2012.

19. Greco SJ, Sarkar S, Casadesus G, Zhu X, Smith MA, Ashford JW, Johnston JM and Tezapsidis N: Leptin inhibits glycogen synthase kinase-3beta to prevent tau phosphorylation in neuronal cells. Neurosci Lett 455: 191-194, 2009.

20. Kim J, Park YJ, Jang Y and Kwon YH: AMPK activation inhibits apoptosis and tau hyperphosphorylation mediated by palmitate in SH-SY5Y cells. Brain Res 1418: 42-51, 2011.

21. Kim B, Figueroa-Romero C, Pacut C, Backus C and Feldman EL: Insulin resistance prevents AMPK-induced tau dephosphorylation through Akt-mediated increase in AMPKSer-485 phosphorylation. J Biol Chem 290: 19146-19157, 2015.

22. Culmsee C,Monnig J, Kemp BE and Mattson MP: AMP-activated protein kinase is highly expressed in neurons in the developing rat brain and promotes neuronal survival following glucose deprivation. J Mol Neurosci 17: 45-58, 2001.

23. Chan KH, Lam KS, Cheng OY, Kwan JS, Ho PW, Cheng KK, Chung SK, Ho JW, Guo VY and Xu A: Adiponectin is protective against oxidative stress induced cytotoxicity in amyloid-beta neurotoxicity. PLoS One 7: e52354, 2012.

24. Dulovic M,Jovanovic M,XilouriM,Stefanis L,Harhaji-Trajkovic L, Kravic-Stevovic T, Paunovic V, Ardah MT, El-Agnaf OM, Kostic V, et al: The protective role of AMP-activated protein kinase in alpha-synuclein neurotoxicity in vitro. Neurobiol Dis 63: 1-11, 2014.

25. Cuesta S, Kireev R, Garcia C, Rancan L, Vara E and Tresguerres JA: Melatonin can improve insulin resistance and aging-induced pancreas alterations in senescence-accelerated prone male mice (SAMP8). Age (Dordr) 35: 659-671, 2013. 
26. Liu HW, Chan YC, Wang MF, Wei CC and Chang SJ: Dietary (-)-epigallocatechin-3-gallate supplementation counteracts aging-associated skeletal muscle insulin resistance and fatty liver in senescence-accelerated mouse. J Agric Food Chem 63: 8407-8417, 2015.

27. Kim HS, Moon S, Paik JH, Shin DW, Kim LS, Park CS, Ha J and Kang JH: Activation of the 5'-AMP-activated protein kinase in the cerebral cortex of young senescence-accelerated P8 mice and association with GSK3 $\beta$ - and PP2A-dependent inhibition of p-tau ${ }_{396}$ expression. J Alzheimers Dis 46: 249-259, 2015.

28. Guo JL, Covell DJ, Daniels JP, Iba M, Stieber A, Zhang B, Riddle DM, Kwong LK, Xu Y, Trojanowski JQ and Lee VM: Distinct $\alpha$-synuclein strains differentially promote tau inclusions in neurons. Cell 154: 103-117, 2013.

29. Giasson BI, Forman MS, Higuchi M, Golbe LI, Graves CL Kotzbauer PT, Trojanowski JQ and Lee VM: Initiation and synergistic fibrillization of tau and alpha-synuclein. Science 300 : 636-640, 2003.

30. Morley JE, Farr SA, Kumar VB and Armbrecht HJ: The SAMP8 mouse: A model to develop therapeutic interventions for Alzheimer's disease. Curr Pharm Des 18: 1123-1130, 2012.

31. de la Monte SM: Brain insulin resistance and deficiency as therapeutic targets in Alzheimer's disease. Curr Alzheimer Res 9: 35-66, 2012

32. Pallàs M,Pizarro JG, Gutierrez-Cuesta J, Crespo-Biel N, Alvira D, Tajes M, Yeste-Velasco M, Folch J, Caudas AM, Sureda FX, et al: Modulation of SIRT1 expression in different neurodegenerative models and human pathologies. Neuroscience 154: 1388-1397, 2008.

33. Long YC, Cheng Z, Copps KD and White MF: Insulin receptor substrates Irs1 and Irs2 coordinate skeletal muscle growth and metabolism via the Akt and AMPK pathways. Mol Cell Biol 31: 430-441, 2011.
34. Burkewitz K, Zhang Y and Mair WB: AMPK at the nexus of energetics and aging. Cell Metab 20: 10-25, 2014.

35. Ruderman NB, Xu XJ, Nelson L, Cacicedo JM, Saha AK, Lan F and Ido Y: AMPK and SIRT1: A long-standing partnership? Am J Physiol Endocrinol Metab 298: E751-E760, 2010.

36. Ruderman NB, Carling D, Prentki M and Cacicedo JM: AMPK, insulin resistance, and the metabolic syndrome. J Clin Invest 123: 2764-2772, 2013.

37. Park H, Kam TI, Kim Y, Choi H, Gwon Y, Kim C, Koh JY and Jung YK: Neuropathogenic role of adenylate kinase-1 in A $\beta$-mediated tau phosphorylation via AMPK and GSK3 $\beta$. Hum Mol Genet 21: 2725-2737, 2012.

38. Hu YY, He SS, Wang X, Duan QH, Grundke-Iqbal I, Iqbal K and Wang J: Levels of nonphosphorylated and phosphorylated tau in cerebrospinal fluid of Alzheimer's disease patients: An ultrasensitive bienzyme-substrate-recycle enzyme-linked immunosorbent assay. Am J Pathol 160: 1269-1278, 2002.

39. Hanger DP, Anderton BH and Noble W: Tau phosphorylation: The therapeutic challenge for neurodegenerative disease. Trends Mol Med 15: 112-119, 2009.

40. Ramamurthy S, Chang E, Cao Y, Zhu J and Ronnett GV: AMPK activation regulates neuronal structure in developing hippocampal neurons. Neuroscience 259: 13-24, 2014.

41. Lin CL, Cheng YS, Li HH, Chiu PY, Chang YT, Ho YJ and Lai TJ: Amyloid- $\beta$ suppresses AMP-activated protein kinase (AMPK) signaling and contributes to $\alpha$-synuclein-induced cytotoxicity. Exp Neurol 275: 84-98, 2016. 